ARTICLE

Received 19 Nov 2012 | Accepted 8 Apr 2013 | Published 14 May $2013 \quad$ DOI: 10.1038/ncomms2842

\title{
Casimir forces on a silicon micromechanical chip
}

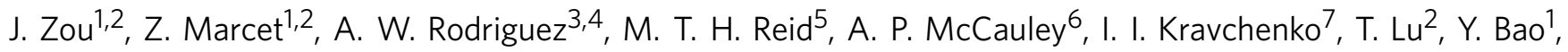
S. G. Johnson ${ }^{4} \&$ H. B. Chan ${ }^{2}$

Quantum fluctuations give rise to van der Waals and Casimir forces that dominate the interaction between electrically neutral objects at sub-micron separations. Under the trend of miniaturization, such quantum electrodynamical effects are expected to play an important role in micro- and nano-mechanical devices. Nevertheless, utilization of Casimir forces on the chip level remains a major challenge because all experiments so far require an external object to be manually positioned close to the mechanical element. Here by integrating a forcesensing micromechanical beam and an electrostatic actuator on a single chip, we demonstrate the Casimir effect between two micromachined silicon components on the same substrate. A high degree of parallelism between the two near-planar interacting surfaces can be achieved because they are defined in a single lithographic step. Apart from providing a compact platform for Casimir force measurements, this scheme also opens the possibility of tailoring the Casimir force using lithographically defined components of non-conventional shapes.

\footnotetext{
${ }^{1}$ Department of Physics, University of Florida, Gainesville, Florida 32611, USA. ${ }^{2}$ Department of Physics, The Hong Kong University of Science and Technology, Clear Water Bay, Kowloon, Hong Kong, China. ${ }^{3}$ School of Engineering and Applied Sciences, Harvard University, Cambridge, Massachusetts 02138, USA. ${ }^{4}$ Department of Mathematics, Massachusetts Institute of Technology, Cambridge, Massachusetts 02139, USA. ${ }^{5}$ Research Laboratory of Electronics, Massachusetts Institute of Technology, Cambridge, Massachusetts 02139, USA. ${ }^{6}$ Department of Physics, Massachusetts Institute of Technology, Cambridge, Massachusetts 02139, USA. ${ }^{7}$ Center for Nanophase Materials Sciences, Oak Ridge National Laboratory, Oak Ridge, Tennessee 37830, USA. Correspondence and requests for materials should be addressed to H.B.C. (email: hochan@ust.hk).
} 
T he Casimir force ${ }^{1}$ refers to the interaction between neutral objects that originates from the boundary conditions imposed on the zero-point quantum fluctuations of the electromagnetic field. Besides fundamental interest, the Casimir force is also of technical importance in micro- and nanomechanical systems, because it is believed to play a role in stiction, which stands for the permanent adhesion of movable components to nearby fixed surfaces ${ }^{2,3}$. Since the first precise measurement of the Casimir force more than a decade ago ${ }^{4}$, significant progress has been made towards the engineering and control of Casimir forces ${ }^{5,6}$. In particular, optical properties and geometry effects provide powerful tools for tailoring the Casimir force. For example, repulsive Casimir forces in the retarded limit were demonstrated in experiments involving fluids ${ }^{7,8}$. Metamaterials, with their remarkable optical properties, have been considered as candidates for controlling the Casimir force ${ }^{9,10}$, but initial suggestions that the sign of the force in vacuum could be altered by metamaterials turned out to be unrealistic $^{11}$. With regard to the non-trivial dependence of the Casimir force on the shape of the bodies ${ }^{12-15}$, experiments involving nanostructured surfaces have demonstrated the nonpairwise additive nature of the Casimir force ${ }^{16,17}$. Recent experiments have also measured the corrections to the Casimir force that arise from the presence of thermal fluctuations, in addition to quantum fluctuations ${ }^{18}$.

The possibility for the Casimir force to play a role in micromechanical systems has been a major driver behind experimental research. However, demonstration of Casimir forces between micromachined surfaces in a single micromechanical chip has remained elusive, because standard experimental schemes require an external object to be manually positioned close to either cantilevers or torsional balances, ${ }^{4,8,19-22}$. Bulky micropositioners and piezoelectric actuators are required to control the separation between the two interacting bodies. Such arrangements have hindered progress in the on-chip exploitation of the Casimir force. Conventional experimental setups also face a number of other challenges. For instance, maintaining the parallelism of two flat surfaces at small distances has proven to be difficult. As a result, in most experiments, one of the two objects is chosen to be spherical. So far, there has only been one experiment that measured the Casimir force between two parallel plates $^{19}$. The alignment becomes even more challenging for nanostructured surfaces. In fact, when corrugations are present on both surfaces, it is necessary to use an in-situ imprint technique such that the patterns are automatically aligned after fabrication ${ }^{17}$. Another major difficulty in measuring the Casimir force at room temperature is the long-term drift in the distance between the surfaces: because the distance from the two interacting elements to their common point of support typically measures at least a few centimeters, temperature fluctuations lead to uncontrollable distance variations, limiting the duration of measurement and hence the force resolution.

We demonstrate that the Casimir force can be the dominant interaction between single-crystalline silicon components on a semiconductor chip, in the absence of external objects. Both the force-sensing element and the actuator that controls the distance are integrated on the same substrate. They are created by dry etching that produces sidewalls that are largely vertical. No external alignment of the interacting bodies is necessary because they are defined in a single lithographic step. The use of electronbeam lithography ensures a high degree of parallelism between the interacting bodies in a near-planar geometry. Another advantage is that the distance of the interacting elements to their common support is reduced to $\sim 70 \mu \mathrm{m}$, about a factor of 1,000 smaller than conventional experiments. The improved mechanical stability minimizes long-term drifts in the gap between the interacting objects $\left(<10^{-5} \mathrm{~nm} \mathrm{~s}^{-1}\right)$. Furthermore, this scheme also allows tailoring of the Casimir force in the future using lithographically defined components of non-conventional shapes.

\section{Results}

Device and measurement scheme. Figure 1a,b shows a simplified schematic of the structure and a scanning electron micrograph of sample A, respectively. The element for sensing the force is a doubly clamped silicon beam that is $100 \mu \mathrm{m}$ long and $\sim 1.42 \mu \mathrm{m}$ wide, depicted in the top parts of Fig. 1a,b. The silicon is p-doped with a high carrier concentration of $7.0 \times 10^{18} \mathrm{~cm}^{-3}$. Figure 1c zooms in on the micro-beam. A silicon electrode of width $2.80 \mu \mathrm{m}$ is positioned close to the beam, as shown in the lower part of Fig. 1c. The beam and the electrode have the same thickness $(2.65 \mu \mathrm{m})$ and distance to the substrate $(2 \mu \mathrm{m})$ (see Methods, Supplementary Fig. S1 and Supplementary Methods for fabrication details). Electrostatic and/or Casimir forces are exerted by the electrode on the beam depending on the voltage $V_{\mathrm{e}}$ between them. The gap between the beam and the electrode is created by deep reactive ion etching (DRIE) while the beam and the electrode are protected with an etch mask. With the etch mask defined by electron-beam lithography, a high degree of parallelism is ensured between the beam and the electrode, without any need for manual alignment before force measurement. By viewing the beam and the electrode from the top with a scanning electron microscope, their separation is found to remain constant to within $\sim 15 \mathrm{~nm}$ along the entire length of the beam, yielding an upper bound of $150 \mu \mathrm{rad}$ for the angle between the lithographic patterns of the beam and the electrode.

The electrode is attached to a comb actuator so that it can be controllably moved along the y direction, reducing the separation $d$ between the electrode and the beam from an initial distance of $d_{0}=1.92 \mu \mathrm{m}$ (measured with a scanning electron microscope) down to $\sim 260 \mathrm{~nm}$ while maintaining parallelism (see Methods, Supplementary Figs S2, S3 and S4, and Supplementary Methods). Figure 1d shows a close-up of part of the comb actuator. The comb actuator consists of a set of movable comb fingers supported by four serpentine springs (Fig. 1e), one at each corner of the structure (Fig. 1a). A second set of comb fingers (the solid structures in Fig. 1d with no etch holes) is fixed to the substrate on one end. When a voltage $V_{\text {comb }}$ is applied to the fixed comb relative to the movable comb (in the experiment, a negative $V_{\text {comb }}$ is used), an electrostatic force parallel to the substrate is generated. The movable combs are displaced towards the beam until the restoring force from the four springs balances the electrostatic force. As a result, the separation $d$ is reduced as $\left|V_{\text {comb }}\right|$ increases (see Supplementary Methods and Supplementary Movies 1 and 2). It should be noted that the electrostatic force between the fixed and movable combs merely serves to set $d$. As explained below, the potential difference between the beam and the movable electrode $V_{\mathrm{e}}$ can be controlled separately, independent of $V_{\text {comb. }}$.

The suspended beam acts as a resonant force sensor. As shown in Fig. 1a, a small alternating voltage $\left(V_{\mathrm{ac}}=5.7 \mu \mathrm{V}\right)$ is applied to one end of the beam, producing an alternating current. In the presence of a $5 \mathrm{~T}$ magnetic field perpendicular to the substrate, the beam is subjected to a periodic Lorentz force. The frequency of the alternating voltage $\omega_{\mathrm{D}}$ is chosen such that the beam vibrates along the $y$ direction in its fundamental mode. Vibrations of the beam in the magnetic field generate an induced electromotive force that is detected with a current amplifier. In Fig. 2a, the resonant frequency of the beam $\omega_{\mathrm{R}}$ is measured to be 7.26185 $\times 10^{6} \mathrm{rad} \mathrm{s}^{-1}$. All measurements were performed at $4 \mathrm{~K}$ and $<10^{-5}$ Torr. When the Casimir force and/or electrostatic forces are exerted on the beam, the resonant frequency $\omega_{R}$ decreases due 

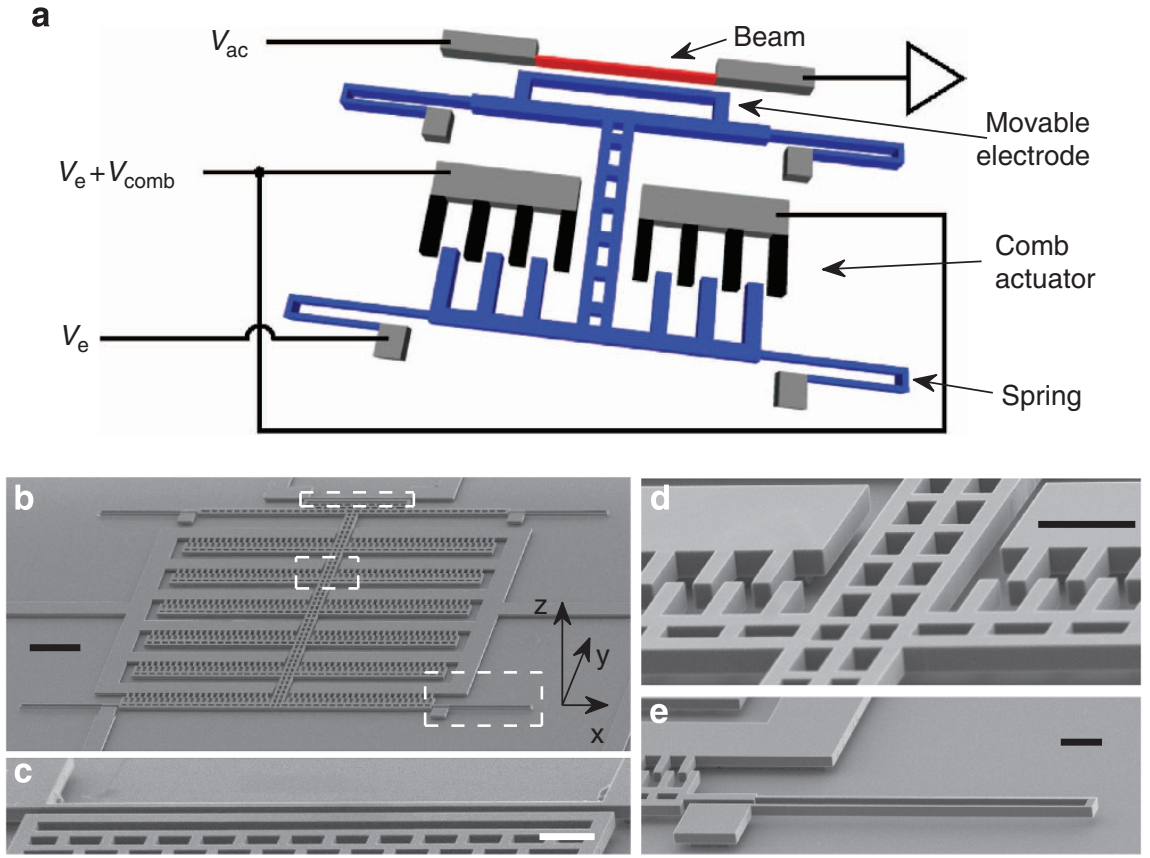

Figure 1 | The setup of the experiment and device. (a) A simplified schematic (not to scale) of the beam (red), movable electrode and comb actuator supported by four springs (blue), with electrical connections. The current amplifier provides a virtual ground to the right end of the beam. Suspended and anchored parts of the comb actuator are shown in blue and dark grey, respectively. The separation $d$ between the beam and the movable electrode was controllably reduced so that the Casimir force can be detected. (b-e) Scanning electron micrographs of the entire micromechanical structure (b) and close-ups of the doubly clamped beam (c), the comb actuator (d) and the serpentine spring (e). The close-ups in c-e zoom into the top, middle and bottom white-dashed boxes in $\mathbf{b}$, respectively. Scale bars, $50 \mu \mathrm{m}$ (b) and $10 \mu \mathrm{m}$ (c-e).

to the spring softening effect, by an amount $\Delta \omega_{\mathrm{R}}$ that is proportional to the force gradient $F^{\prime}(d)$ :

$$
\Delta \omega_{\mathrm{R}}=K F^{\prime}(d),
$$

where $K$ is a positive proportionality constant and $F^{\prime}(d)<0$.

Calibration. Similar to conventional experiments on Casimir forces, we also need to perform a calibration procedure by applying a voltage $V_{\mathrm{e}}$ between the beam and the movable electrode to generate an electrostatic force $F_{\mathrm{e}}$ between them. $F_{\mathrm{e}}$ is proportional to $\left(V_{\mathrm{e}}-V_{0}\right)^{2}$, where $V_{0}$ is the residual voltage. Figure $2 \mathrm{~b}$ shows parabolic fits to $\Delta \omega_{\mathrm{R}}$ versus $V_{\mathrm{e}}$. Each parabola corresponds to $\Delta \omega_{\mathrm{R}}$ recorded at a fixed $d$ that is set by $V_{\text {comb. }}$ There are two contributions to $\Delta \omega_{\mathrm{R}}$ : the electrostatic part that depends quadratically on $V_{\mathrm{e}}-V_{0}$ and a vertical offset that is independent of $V_{\mathrm{e}}-V_{0}$. The latter becomes more negative as $d$ decreases. As described later, we will compare this vertical offset to the Casimir force gradient and remnant force gradients due to patch potentials. The electrostatic part will be used for force calibration.

The residual voltage $V_{0}$ is measured by identifying $V_{\mathrm{e}}$ at which the maximum of the parabolic dependence of $\Delta \omega_{\mathrm{R}}$ occurs. Figure $2 \mathrm{c}$ shows that $V_{0}$ is measured to be about $-25 \mathrm{mV}$ at small $d$. Over the full range of distances, $V_{0}$ changes by about $15 \mathrm{mV}$, comparable to previous experiments in the lens-plate ${ }^{23}$ and sphere-plate ${ }^{24}$ geometries. Even though both the beam and the electrode are made of single-crystal silicon on the same wafer, the residual voltage $V_{0}$ is non-zero and shows distance dependence. Based on current experimental data, we cannot convincingly identify the origin of these effects. We suspect that the non-zero residual voltage is possibly due to solder contacts in the electrical leads at different temperatures. The distance dependence likely originates from adsorbed impurities and/or the etching profile (see Supplementary Methods) exposing patches of different crystal orientations at the sidewalls with non-uniform potentials.

In conventional Casimir force experiments, the extension of the piezoelectric element is either pre-calibrated or directly measured. At the same time, the initial distance between the two interacting surfaces is an unknown that needs to be determined by the application of electrostatic forces. In our experiment, distance calibration is performed using a slightly different procedure. Here $d$ is given by:

$$
d=d_{0}-\alpha V_{\mathrm{comb}}^{2},
$$

where $d_{0}$ is the initial separation, $V_{\text {comb }}$ is the voltage of the fixed combs relative to the movable combs and $\alpha=\left(\mathrm{d} C_{\text {comb }} / \mathrm{d} y\right) / 2 k_{/}$ is a proportionality constant to be determined by fitting. $k_{/ /}$is the spring constant of the serpentine springs along $y . \mathrm{d} C_{\text {comb }} / \mathrm{d} y$, the spatial derivative of the capacitance between the fixed and the movable combs, remains almost constant as the movable combs are displaced. One main difference from previous experiments is that $d_{0}$ is not a fitting parameter. Instead, $d_{0}$ is accurately measured to be $1.92 \pm 0.015 \mu \mathrm{m}$ using a scanning electron microscope. The dependence of $F_{\mathrm{e}}$ on the distance $d$ is calculated using finite element analysis with our device geometry. By fitting to the calculated electrostatic force gradient $F_{\mathrm{e}}^{\prime}(d)$ given by equations (1) and (2) for six sets of data with $V_{\mathrm{e}}$ ranging from $V_{0}+100$ to $V_{0}+150 \mathrm{mV}$, we obtain $\alpha=10.55 \pm 0.04 \mathrm{~nm} \mathrm{~V}^{-2}$ and $K=5.38 \pm 0.10 \times 10^{4} \mathrm{radm}(\mathrm{s} \mathrm{N})^{-1}$. Figure $2 \mathrm{~d}$ plots the electrostatic force gradient on the beam as a function of $d$ at $V_{\mathrm{e}}=V_{0}+100 \mathrm{mV}$, where $d$ is controlled by increasing $\left|V_{\text {comb }}\right|$ from 0 to $11.375 \mathrm{~V}$ according to equation (2). When $V_{\mathrm{e}}$ is applied, the effective distance for the electrostatic force changes because of carrier depletion. However, because of the high carrier concentration $\left(7 \times 10^{18} \mathrm{~cm}^{-3}\right)$, this change is small $(<1 \mathrm{~nm})$ and negligible compared with the uncertainty in $d$. 
a

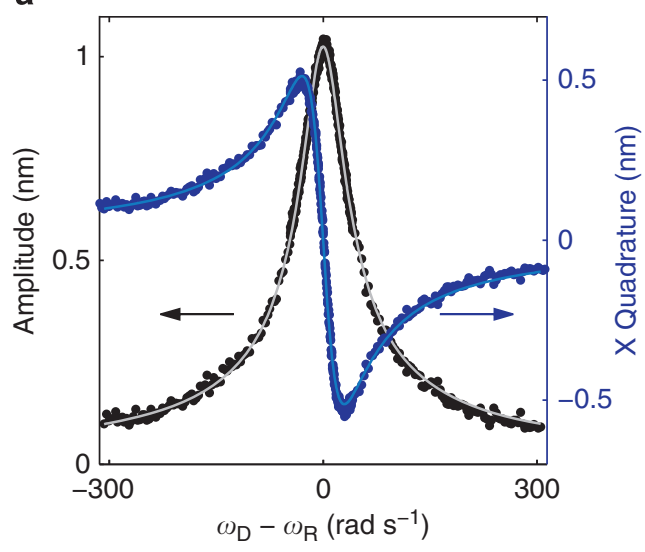

C

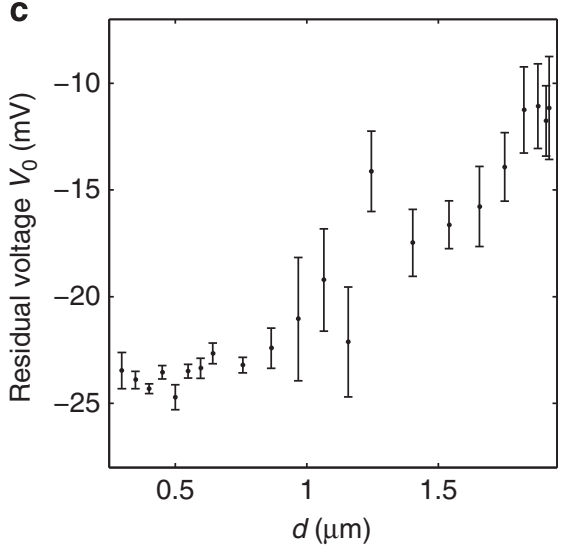

b

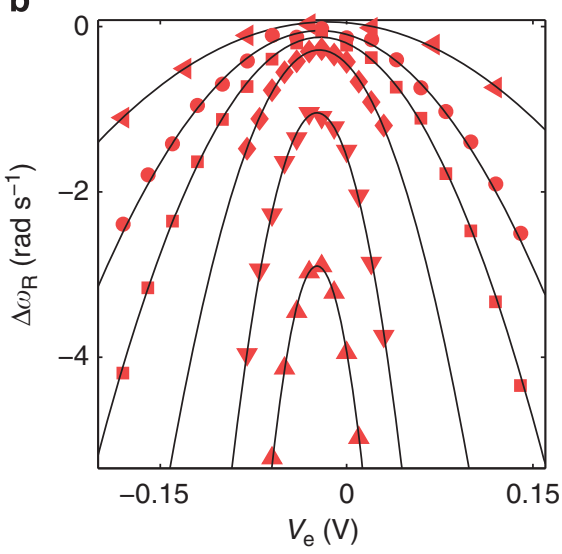

d

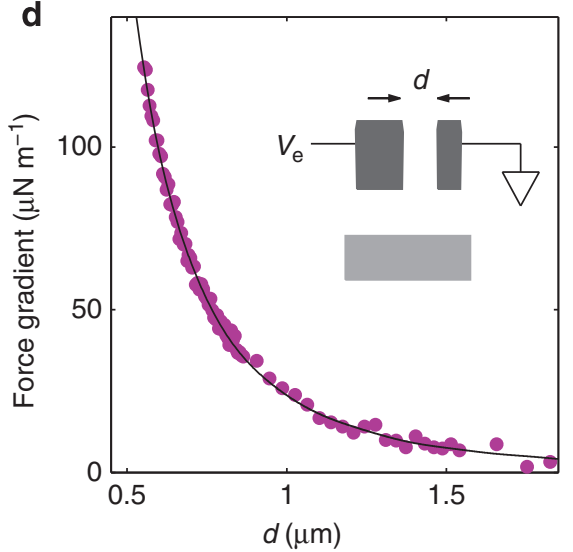

Figure 2 | Calibration of the device using electrostatic force gradient. (a) Oscillation amplitude of the beam and its $X$ quadrature that is in phase with the periodic driving force. Fitting to the driven underdamped oscillator model (lines) gives a damping coefficient of 30 rad s $^{-1}$. (b) Measured frequency shift $\Delta \omega_{\mathrm{R}}$ as a function of electrode voltage $V_{\mathrm{e}}$, at $d=1.403 \mu \mathrm{m}, 1.065 \mu \mathrm{m}, 865 \mathrm{~nm}, 643 \mathrm{~nm}, 450 \mathrm{~nm}$ and $349 \mathrm{~nm}$, from top to bottom. (c) Measured dependence of the residual voltage as a function of $d$. (d) Measured electrostatic force gradient on the beam (circles) at $V_{\mathrm{e}}=V_{0}+100 \mathrm{mV}$. The line represents a fit to the values calculated using finite element analysis. Inset: cross-sectional schematic of the beam, electrode and substrate with direct current (dc) electrical connections. Error bars represent \pm 1 s.e.

Casimir force measurement and calculations. Next, we set $V_{\mathrm{e}}=V_{0}(d)$ for each distance $d$ and measure the force gradient $F_{\mathrm{c}}^{\prime}$ between the beam and the electrode as a function of $d$ (Fig. 3a). The red line in Fig. 3a represents the theoretical values of the Casimir force calculated for silicon structures of such geometry, with no fitting parameters. The theoretical calculation involves a boundary-element method (BEM) discretization of the beam and substrate surfaces, combined with a recent fluctuating-surfacecurrent formulation of the Casimir force between dielectric bodies that writes the full Casimir-energy path integral as a simple expression in the classical BEM interaction matrix ${ }^{14,25}$. It includes the contributions of the finite conductivity of silicon and the imperfect etching profiles on the sidewalls of the beam and the electrode ( $\sim 88^{\circ}$ from the substrate surface, see Supplementary Methods). Despite the imperfect agreement between measurement and theory, it is clear that the Casimir force becomes the dominant interaction between the beam and the movable electrode at small $d$. Unlike the sphere-plate configuration, the roughness on the sidewalls cannot be directly measured. From the top-view micrograph of the beam and electrode, we determine the root-mean-square ( $\mathrm{rms}$ ) roughness of the edges to be $12 \mathrm{~nm}$, mainly due to non-uniformity of the electron-beam lithography. The roughness correction is estimated to be about $3 \%$ of the Casimir force at the closest distance ${ }^{26}$. We further note that the calculation assumes the lithographic patterns of the beam and the electrode to be parallel. Using an upper bound of $150 \mu \mathrm{rad}$ for the angle between them, the calculated Casimir force increases by up to $1.1 \%$ at the closest distance ${ }^{26}$.

One plausible explanation for the deviations involves residual electrostatic forces that are associated with patch potentials. Following the analysis of Kim et al. ${ }^{23}$, such residual forces display similar distance dependence as the applied electrostatic force, but varies as $\left(V_{0}(d)+V_{1}\right)^{2}+V_{\mathrm{rms}}^{2}$. The first term originates from the spatial variations in the contact potential that give rise to a distance-dependent $V_{0}$, whereas the second term accounts for patches smaller than the effective interaction area. $V_{1}$ accounts for a constant offset of the averaged contact voltage at large distances, whereas $V_{\text {rms }}$ is the rms value of the random patch voltages. Currently, our experiment lacks the sensitivity to resolve the force gradients at $d>0.8 \mu \mathrm{m}$. Therefore, unlike Kim et al. ${ }^{23}$, we cannot measure long-range residual electrostatic forces and fit at distances where the Casimir force is negligible. Instead, we fit the measured force gradients to the calculated Casimir force gradient modified by residual electrostatic forces due to patch potentials (purple line in Fig. 3a). By setting $V_{1}=-V_{0}=11.3 \mathrm{mV}$ at the largest $d$, we obtain a fitted value of $V_{\mathrm{rms}}=16.8 \mathrm{mV}$.

\section{Discussion}

The Casimir force gradient between a beam and an electrode with near-square cross-sections has not been measured experimentally before. This configuration opens the possibility of testing a 


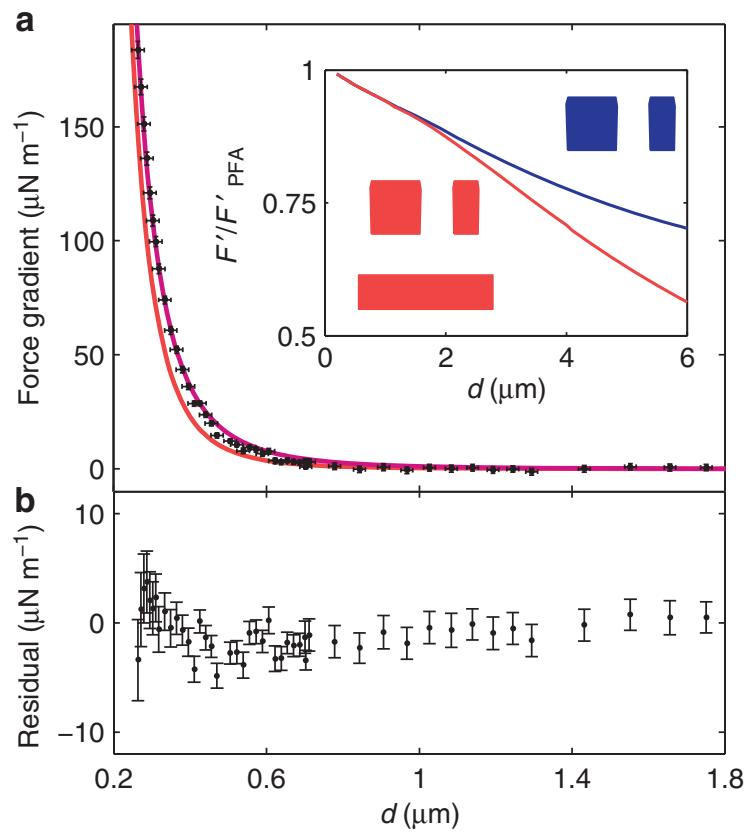

Figure 3 | Measured force gradient $F^{\prime}{ }_{c}$ between the beam and the movable electrode as a function of separation $d$ after compensating for the residual voltage. (a) The red line represents the calculated Casimir force gradients between an electrode and a beam made of silicon. The purple line includes possible contributions from patch potentials. Inset: the ratios of the calculated Casimir force between the beam and the electrode to the forces given by the PFA are plotted as the red (with substrate) and blue lines (without substrate). (b) Deviations of the measured force gradient from the purple line in $\mathbf{a}$. Error bars represent \pm 1 s.e.

number of fundamental concepts. One important question is the validity of the proximity force approximation (PFA ${ }^{27}$. The inset of Fig. 3a compares the Casimir force gradient generated by the PFA with calculations of the exact geometry of the silicon structures. The ratio decreases with distance, reaching 56\% at $6 \mu \mathrm{m}$. Second, this geometry could offer a direct demonstration of the non-pairwise nature of the Casimir force. The inset of Fig. $3 \mathrm{a}$ shows that the calculated Casimir force between the beam and the electrode depends on the presence of a third body, the substrate. When the substrate is removed, the Casimir force increases by $\sim 14 \%$ at $6 \mu \mathrm{m}$. We note that the PFA yields the same force on the beam regardless of whether a substrate is present, as interactions with the third body are not taken into account. Recent theoretical analysis also predicted that the Casimir force between the beams exhibits a non-monotonic dependence on the distance to the substrate that cannot be explained by pairwise additive models of the force ${ }^{28}$. Because the Casimir force gradient at these distances is beyond the reach of the current setup, future experiments to reveal the aforementioned effects would require more sophisticated measurement circuitry or other detection schemes ${ }^{29-31}$ to improve the sensitivity at large $(>2 \mu \mathrm{m})$ separations. Alternatively, beams and electrodes with smaller cross-sections and/or smaller beam-substrate separations can be used to generate Casimir forces that are distinguishable from the PFA and pairwise additivity at smaller distances. Further progress will also require a better understanding of the force from the patches potentials, to suppress such forces or separate them from the Casimir force in the total measured force.

We have shown that the Casimir force can be the dominant interaction between silicon micromechanical components on an integrated micromechanical chip, in the absence of external objects. By creating the two interacting surfaces using nanofabrication, we circumvented the problem of aligning two planar surfaces. Our results represent the first step towards on-chip exploitation of the Casimir force. The compact and integrated actuation and detection platform provides new opportunities of tailoring the Casimir force using geometry effects. Structures are defined by electron-beam lithography, making it possible to create surfaces with complex shapes 32 that are automatically aligned after fabrication. Furthermore, the design can be integrated with suspended planar waveguide elements so that classical optical gradient forces ${ }^{31}$ and Casimir forces associated with virtual photons can be simultaneously utilized in these optomechanical systems. Nevertheless, much progress will be necessary, particularly in sample fabrication and characterization, to achieve the $<5 \%$ agreement between theory and measurement ${ }^{18,21,22}$ that has been claimed in conventional Casimir force experiments between spheres and plates.

\section{Methods}

Device fabrication and preparation. The device is fabricated using a silicon-oninsulator wafer with a $2.65-\mu \mathrm{m}$ silicon device layer and a $2.0-\mu \mathrm{m}$ buried oxide layer, so that the beam, electrode and comb actuator have identical thickness and distance from the substrate. The gap between the beam and the plate is created by DRIE of the silicon device layer while the beam and the electrode are protected with an etch mask defined by electron-beam lithography. After removing the etch mask and the underlying sacrificial silicon-oxide layer with hydrofluoric acid (HF), the beam is free to vibrate in response to external forces (see Supplementary Methods).

Both the device layer and the handle wafer are p-doped with boron. The resistivity and carrier concentration of the device layer are measured to be $0.011 \Omega \mathrm{cm}$ and $7.0 \times 10^{18} \mathrm{~cm}^{-3}$, respectively, at $4 \mathrm{~K}$ by the van der Pauw method. For the handle wafer, the resistivity is measured to be $21.5 \Omega \mathrm{cm}$ at room temperature. We find that it becomes effectively insulating at $4 \mathrm{~K}$.

To avoid charges being trapped on the surfaces of the beam and the movable electrode, HF is used to remove the native oxide on silicon right before force measurement. HF also passivates the surfaces to temporarily prevent the reformation of oxide at ambient pressure ${ }^{16}$. The chip is then immediately loaded into a sealed probe that is evacuated and then lowered into a cryostat.

Measurement of the force gradient on the beam. The shift $\Delta \omega_{\mathrm{R}}$ is measured by maintaining the alternating voltage at a fixed frequency and recording the change in the $\mathrm{X}$ quadrature. Provided that $\omega_{\mathrm{R}}$ remains close to the frequency of the alternating voltage, small changes in the $\mathrm{X}$ quadrature is proportional to $\Delta \omega_{\mathrm{R}}$, which is in turn proportional to $F^{\prime}(d) . \Delta \omega_{\mathrm{R}}$ can be inferred using the change in the $\mathrm{X}$ quadrature and the slope of the $\mathrm{X}$ quadrature response (the blue curve in Fig. 2a). At the largest measured $\Delta \omega_{\mathrm{R}}$, we estimate the deviation from a linear relationship between the X quadrature and $\Delta \omega_{\mathrm{R}}$ to be $<2.5 \%$, which is smaller than our measurement uncertainty. The oscillation amplitude of the beam is kept small $(<1.4 \mathrm{~nm}<<d)$. We calibrate the oscillation amplitude of the beam by measuring the onset of nonlinear mechanical bistability. Static deflection of the beam in response to the electrostatic and Casimir forces from the movable electrode is $<2 \mathrm{pm}$, negligible compared with $d$. We do not resolve any change in the width of the amplitude response (Fig. 2a) of the beam with distance.

Geometry characterization. As described in the main text, the nanofabrication process produces two interacting surfaces that are automatically aligned and almost parallel to each other. Deviations from the ideal parallel-plane geometry may occur due to imperfections in the processing steps. For example, drift and misalignment of the electron-beam writer may produce a lithographic pattern that deviates from perfect parallelism. Using scanning electron microscopy, we analysed the close-up top views of the gap close to the two ends of the beam and at different locations of the beam. Within the measurement uncertainty, we did not observe any change of the gap across the beam. We estimate that the upper bound for the angle between the lithographic patterns of the beam and the electrode is $150 \mu \mathrm{rad}$ (corresponding to a gap changing $15 \mathrm{~nm}$ over $100 \mu \mathrm{m}$ ). Using the above parameters, the force increases by $<1.1 \%$ at the smallest separation.

For a structure with dimensions exactly equal to the nominal values in the electron-beam lithographic pattern, the angle between the beam and the movable electrode remains unchanged as $d$ decreases. In the actual device, however, nonuniformities in exposure and etching might produce small asymmetries in the structure. To estimate such effects, we consider the case in which the two springs on the left side is wider than the ones on the right side by $50 \mathrm{~nm}$. This value is a conservative estimate because it significantly exceeds the resolution of the electronbeam writer. Numerical calculations show that at the largest $\left|V_{\text {comb }}\right|$, the movable 
electrode is closer to the beam by $<4 \mathrm{~nm}$ on the right side than the left side. This value is smaller than the roughness of the sidewalls of the beam and the movable electrode and leads to a negligible tilting angle $\sim 40 \mu \mathrm{rad}$. Our calculation indicates that it increases the Casimir force by $<0.1 \%$ at the closest distance.

The cross-sectional profiles of the beam and the electrode were also analysed, by cleaving a second sample that is fabricated together with the sample in which the Casimir force was measured (Supplementary Fig. S3). The slightly convex slope near the top of the cross-section arises because of the periphery of the aluminium etch mask being consumed in the dry etching process. For the rest of the sidewall, the DRIE recipe produces a concave profile at an angle of $\sim 88^{\circ}$ to the top surface. This geometry was used in the BEM numerical calculation and the PFA. More details are discussed in Supplementary Methods.

Calculations of the Casimir force. The theoretical calculation (red line in Fig. 3a) neglects the finite length of the beams. The geometry reduces to a two-dimensional problem in the cross-sections (integrated over the longitudinal wavevector), and each object's surface is discretized into set of line segments described by 'rooftop' basis functions ${ }^{25}$. We found that a discretization of approximately 3,200 total points for all surfaces and a substrate truncated to $1 \mu \mathrm{m}$ was sufficient to obtain convergence to $1 \%$ accuracy.

In the calculations, the dielectric function of silicon $\varepsilon(i \xi)$ is obtained using the Kramers-Kronig relations with the tabulated optical constants for frequency $\xi>5,000 \mathrm{rad} \mathrm{s}^{-1}$. For $\xi<5,000 \mathrm{rad} \mathrm{s}^{-1}, \varepsilon(i \xi)$ is given by:

$$
\varepsilon(i \xi)=1.0035+(11.87-1.0035) /\left(1+\xi^{2} / \omega_{0}^{2}\right)+\omega_{\mathrm{p}}^{2} /[\xi(\xi+\Gamma)],
$$

where $\omega_{0}=6.6 \times 10^{15} \mathrm{rad} \mathrm{s}^{-1}, \omega_{\mathrm{p}}=2.5562 \times 10^{14} \mathrm{rad} \mathrm{s}^{-1}$ and

$\Gamma=6.364 \times 10^{13} \mathrm{rad} \mathrm{s}^{-1}$.

In estimating the roughness correction, the force between two flat plates with stochastic roughness is first calculated using equation (16) in Bordag et al. ${ }^{26}$ as a function of distance. Then, the PFA is used to generate the force for our geometry.

\section{References}

1. Casimir, H. B. G. On the attraction between two perfectly conducting plates. Proc. Kon. Ned. Akad. Wet. 51, 793-795 (1948).

2. Serry, F. M., Walliser, D. \& Maclay, G. J. The Anharmonic Casimir Oscillator (ACO) - the Casimir effect in a model microelectromechanical system. $J$. Microelectromech. Syst. 4, 193-205 (1995).

3. Buks, E. \& Roukes, M. L. Metastability and the Casimir effect in micromechanical systems. Europhys. Lett. 54, 220-226 (2001).

4. Lamoreaux, S. K. Demonstration of the Casimir force in the 0.6 to $6 \mu \mathrm{m}$ range. Phys. Rev. Lett. 78, 5-8 (1997).

5. Klimchitskaya, G. L., Mohideen, U. \& Mostepanenko, V. M. The Casimir force between real materials: Experiment and theory. Rev. Mod. Phys. 81, 1827-1885 (2009).

6. Rodriguez, A. W., Capasso, F. \& Johnson, S. G. The Casimir effect in microstructured geometries. Nat. Photonics 5, 211-221 (2011).

7. Dzyaloshinskii, I. E., Lifshitz, E. M. \& Pitaevskii, L. P. The general theory of van der Waals forces. Adv. Phys. 10, 165-209 (1961).

8. Munday, J. N., Capasso, F. \& Parsegian, V. A. Measured long-range repulsive Casimir-Lifshitz forces. Nature 457, 170-173 (2009).

9. Leonhardt, U. \& Philbin, T. G. Quantum levitation by left-handed metamaterials. N. J. Phys. 9, 254 (2007).

10. Zhao, R., Zhou, J., Koschny, T., Economou, E. N. \& Soukoulis, C. M. Repulsive Casimir force in chiral metamaterials. Phys. Rev. Lett. 103, 103602 (2009).

11. McCauley, A. P. et al. Microstructure effects for Casimir forces in chiral metamaterials. Phys. Rev. B 82, 165108 (2010).

12. Lambrecht, A., Neto, P. A. M. \& Reynaud, S. The Casimir effect within scattering theory. N. J. Phys. 8, 243 (2006).

13. Rahi, S. J., Emig, T., Graham, N., Jaffe, R. L. \& Kardar, M. Scattering theory approach to electrodynamic Casimir forces. Phys. Rev. D 80, 085021 (2009).

14. Reid, M. T. H., Rodriguez, A. W., White, J. \& Johnson, S. G. Efficient computation of Casimir interactions between arbitrary 3D objects. Phys. Rev. Lett. 103, 040401 (2009).

15. Davids, P. S., Intravaia, F., Rosa, F. S. S. \& Dalvit, D. A. R. Modal approach to Casimir forces in periodic structures. Phys. Rev. A 82, 062111 (2010).

16. Chan, H. B. et al. Measurement of the Casimir force between a gold sphere and a silicon surface with nanoscale trench arrays. Phys. Rev. Lett. 101, 030401 (2008).

17. Chiu, H. C., Klimchitskaya, G. L., Marachevsky, V. N., Mostepanenko, V. M. \& Mohideen, U. Lateral Casimir force between sinusoidally corrugated surfaces:
Asymmetric profiles, deviations from the proximity force approximation, and comparison with exact theory. Phys. Rev. B 81, 115417 (2010).

18. Sushkov, A. O., Kim, W. J., Dalvit, D. A. R. \& Lamoreaux, S. K. Observation of the thermal Casimir force. Nat. Phys. 7, 230-233 (2011).

19. Bressi, G., Carugno, G., Onofrio, R. \& Ruoso, G. Measurement of the Casimir force between parallel metallic surfaces. Phys. Rev. Lett. 88, 041804 (2002).

20. Chan, H. B., Aksyuk, V. A., Kleiman, R. N., Bishop, D. J. \& Capasso, F. Quantum mechanical actuation of microelectromechanical systems by the Casimir force. Science 291, 1941-1944 (2001).

21. Decca, R. S. et al. Constraining new forces in the Casimir regime using the isoelectronic technique. Phys. Rev. Lett. 94, 240401 (2005).

22. Mohideen, U. \& Roy, A. Precision measurement of the Casimir force from 0.1 to $0.9 \mu \mathrm{m}$. Phys. Rev. Lett. 81, 4549-4552 (1998).

23. Kim, W. J., Brown-Hayes, M., Dalvit, D. A. R., Brownell, J. H. \& Onofrio, R. Anomalies in electrostatic calibrations for the measurement of the Casimir force in a sphere-plane geometry. Phys. Rev. A 78, 020101 (2008).

24. Torricelli, G. et al. Switching Casimir forces with phase-change materials. Phys Rev. A 82, 010101 (2010).

25. Reid, M. T. H. Fluctuating Surface Currents: A New Algorithm for Efficient Prediction of Casimir Interactions among Arbitrary Materials in Arbitrary Geometries. Thesis. Massachusetts Institute of Technology (2010).

26. Bordag, M., Klimchitskaya, G. L. \& Mostepanenko, V. M. Corrections to the Casimir Force between plates with stochastic surfaces. Phys. Lett. A 200, 95-102 (1995).

27. Derjaguin, B. V. Direct measurement of molecular attraction between solids separated by a narrow gap. Q. Rev. Chem. Soc. 10, 295-329 (1956).

28. Rodriguez, A. et al. Computation and visualization of Casimir forces in arbitrary geometries: Nonmonotonic lateral-wall forces and the failure of proximity-force approximations. Phys. Rev. Lett. 99, 080401 (2007).

29. Faust, T., Krenn, P., Manus, S., Kotthaus, J. P. \& Weig, E. M. Microwave cavityenhanced transduction for plug and play nanomechanics at room temperature. Nat. Commun. 3, 728 (2012).

30. Li, M., Pernice, W. H. P. \& Tang, H. X. Tunable bipolar optical interactions between guided lightwaves. Nat. Photonics 3, 464-468 (2009).

31. Van Thourhout, D. \& Roels, J. Optomechanical device actuation through the optical gradient force. Nat. Photonics 4, 211-217 (2010).

32. Rodriguez, A. W., Joannopoulos, J. D. \& Johnson, S. G. Repulsive and attractive Casimir forces in a glide-symmetric geometry. Phys. Rev. A 77, 062107 (2008).

\section{Acknowledgements}

H.B.C., J.Z., Z.M. and Y.B. are supported by DOE No. DE-FG02-05ER46247 and NSF No. DMR-0645448. H.B.C. and T.L. are supported by Shun Hing Solid State Clusters Lab and HKUST 600511 from the Research Grants Council of Hong Kong SAR. S.G.J., A.W.R. and A.P.M are supported in part by DARPA under contract N66001-09-1-2070DOD. M.T.H.R. was supported by the Singapore-MIT Alliance's Program in Computational Engineering. A portion of this research was conducted at the Center for Nanophase Materials Sciences, which is sponsored at Oak Ridge National Laboratory by the Office of Basic Energy Sciences, US Department of Energy.

\section{Author contributions}

J.Z. and H.B.C. conceived the experiment. J.Z., Z.M., I.I.K. and Y.B. designed, fabricated and characterized the devices. Z.M. and J.Z. conducted the measurements. Z.M., J.Z., T.L. and H.B.C. analysed the data. A.W.R., M.T.H.R., A.P.M. and S.G.J. performed the theoretical calculations. H.B.C. supervised the experiment. All authors discussed the result and contributed to the writing.

\section{Additional information}

Supplementary Information accompanies this paper at http://www.nature.com/ naturecommunications

Competing financial interests: The authors declare no competing financial interests

Reprints and permission information is available online at http://npg.nature.com/ reprintsandpermissions/

How to cite this article: Zou, J. et al. Casimir forces on a silicon micromechanical chip. Nat. Commun. 4:1845 doi: 10.1038/ncomms2842 (2013). 